Beobachtungen des Cometen 1893... (Rordame-Quénisset)

angestellt auf der Sternwarte der meteorologischen Central-Anstalt zu Budapest.

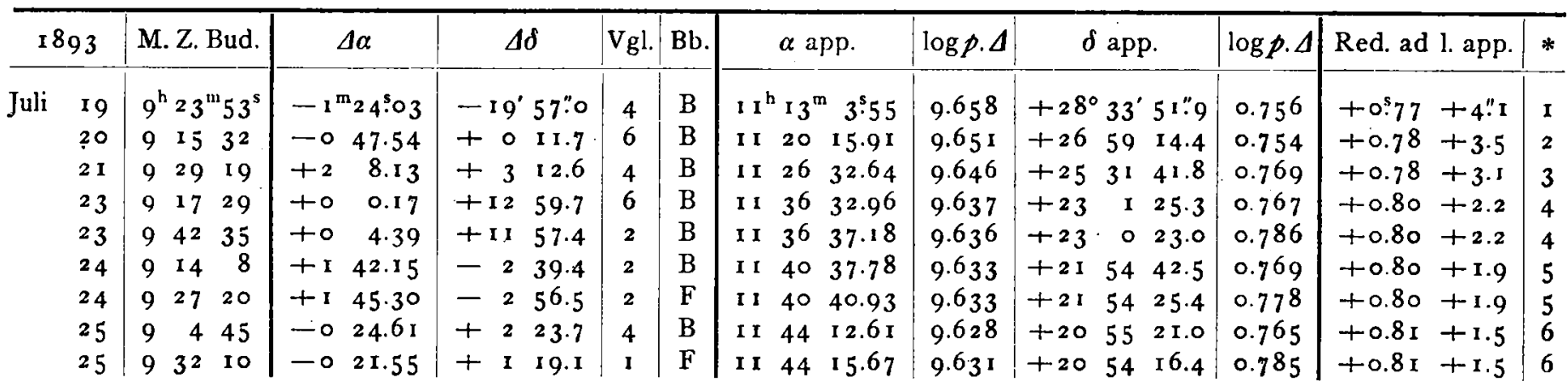

Instrument : $4 \frac{1}{2}$ Zöller von Merz.

Beobachter: $\mathbf{B}=$ Dr. Buschbaum; $\mathbf{F}=$ L. Fraunhoffer, Assistent an der meteor. Central-Anstalt.

Juli r 9. Comet erscheint als runder Nebel mit hellem Kern. - Juli 23. Erste Beobachtung weniger gut, da sich Vergleichstern und Comet nahezu auf demselben Stundenkreis befinden und daher die Beobachtung erschwert wird. - Juli 24. Comet schwächer als in den vorangehenden Tagen.

Die geogr. Länge der Sternwarte ist nach der Angabe in den »Beobachtungen am astrophysikalischen Observatorium zu O-Gyalla (Band XIII u. XIV) $22^{\mathrm{m}} 33^{\mathrm{s}} \cdot 05$ östlich von Berlin.

Mittlere Oerter der Vergleichsterne für 1893.0 .

\begin{tabular}{|c|c|c|c|}
\hline$*$ & $\alpha$ & $\delta$ & Autorität \\
\hline $\mathbf{I}$ & $1 x^{h} 14^{m} 26.81$ & $+28^{\circ} 53^{\prime} 44^{\prime \prime} 8$ & $\mathrm{Par}_{2}$ I $_{3829}$ \\
\hline 2 & I I $21 \quad 2.67$ & +265859.2 & $W_{2}=1.347$ \\
\hline 3 & $\begin{array}{lll}x & 24 & 23.73\end{array}$ & +252826.1 & $\mathrm{Par}_{2}$ I 4044 \\
\hline
\end{tabular}

\begin{tabular}{|c|c|c|c|}
\hline * & $\alpha$ & $\delta$ & Autorität \\
\hline 4 & $\operatorname{lr}^{\mathrm{h}} 3^{6^{\mathrm{m}}} 3^{1}{ }^{\mathrm{s}} \cdot 99$ & $+22^{\circ} 4^{\prime} 23^{\prime \prime} 4$ & $1 / 2\left(\operatorname{Par}_{2} 1_{4} 287+\operatorname{Arm}_{2}{ }_{34}\right.$ \\
\hline 5 & I I $38 \quad 54.83$ & +215720.0 & BB. VI $+22: 2396$ \\
\hline 6 & I I $44 \quad 36.41$ & +205255.8 & $W_{2} \times 1$ h $841-42$ \\
\hline
\end{tabular}

Budapest 1893 August.

C. Buschbaum.

\title{
Osservazioni della Cometa 1893 ... (Rordame-Quénisset)
}

fatte a Padova coll' equatoriale Dembowski.

Obbiettivo $187 \mathrm{~mm}$. Nuovo micrometro a lamine del passo angolare di $\mathbf{3}^{2 !} \mathbf{3}$.

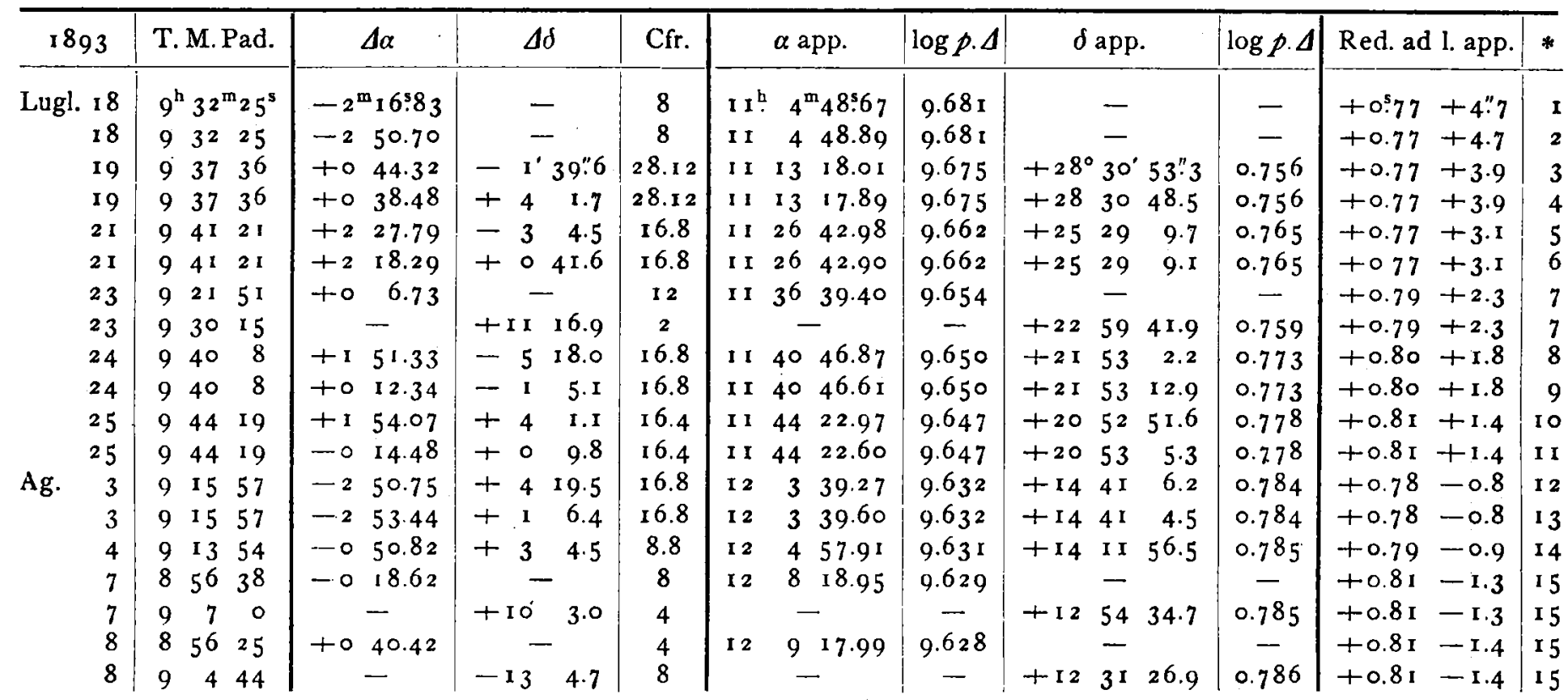


Luglio 18 . La continua variabilità del cielo all' orizzonte ovest, dove stava la Cometa prossirma a tramontare, non permise di vederla prima di questa sera nella quale le osservazioni riuscirono incomplete per la stessa ragione, cioè del nuvolo sopravveniente. - Luglio r9. Sereno bellissimo tutta la notte. La Cometa apparve come un bel stellone nebuloso del diametro di $2^{\prime}$ circa, con traccia leggerissima di coda seguente la testa nella direzione del parallelo. Fu vista ad occhio nudo e fu stimata meno splendida delle due stelle prossime, $\xi$ e $\boldsymbol{\nu}$ della zampa dell' orsa maggiore. - Luglio 2 I e 23 . Sereno fosco ed infine nuvolo. Osservazioni poco soddisfacenti. - Luglio 24 e 25. Bel sereno ma chiaro di luna. Gli ostacoli terrestri limitano ancora di più il breve tempo in cui la cometa è visibile prima del suo tramonto che avviene poco dopo il crepuscolo. - Agosto 3. Sereno splendido. Cometa affievolita. - Agosto 4 e 7 . Sereno fosco. Osservata molto bassa ed incompletamente causa la sua sparizione dietro i tetti delle case situate all ovest del Dembowski.

Agosto 8. Bel sereno. Osservazioni e scarse ed incomplete per essere la cometa bassissima.

Stelle di confronto pel $1893,0$.

\begin{tabular}{|c|c|c|c|c|c|c|}
\hline * & \multicolumn{2}{|r|}{$\alpha$} & \multicolumn{3}{|c|}{$d$} & Autorità \\
\hline $\mathbf{I}$ & $\mathrm{II}^{\mathrm{h}}$ & $7^{m} \quad 4^{5} \cdot 73$ & $+30^{\circ}$ & & 19"7 & Leid. AG. Z. 22, 24 \\
\hline 2 & II & 738.82 & +30 & 8 & $7 \cdot 5$ & Leid. AG. Z. 22,24 \\
\hline 3 & II 1 & 232.92 & +28 & $3^{2}$ & 29.0 & BB.VI $+28^{\circ} 19^{8} 5$ \\
\hline 4 & I I I & $23^{8.64}$ & +28 & 26 & 42.9 & BB.VI + 28.1986 \\
\hline 5 & II 2 & $4 \times 4.42$ & +25 & $3^{2}$ & I I. I & $W_{2} I 1^{\mathrm{b}} \cdot 4 \mathrm{I}_{2}$ \\
\hline 6 & I I 2 & $4 \quad 23.84$ & +25 & 28 & 24.4 & $W_{2} I^{h} \cdot 4 I 4$ \\
\hline 7 & I 3 & 631.88 & +22 & 48 & 22.7 & Berl. AG. Z 68, 23 \\
\hline 8 & II 3 & $8 \quad 54.74$ & $+2 I$ & $5^{8}$ & I 8.4 & Berl. AG. Z. 5 I, 55 \\
\hline
\end{tabular}

$$
\begin{aligned}
& \text { * I. } \mathrm{W}_{2} \text { II } \mathrm{r}^{\mathrm{h}} \mathrm{g}_{3} \text { - Leid. AG. Z. }=-0.53+6.8 \text {. }
\end{aligned}
$$



$$
\begin{aligned}
& \text { * 7. W } \mathrm{W}_{2} 677.679 \text { - Berl. AG.Z. }=+0.35+2.5 \\
& \mathrm{Y}_{3}-\text { Berl. AG. Z. }=+0.08+1: 3 \\
& \mathrm{Arm}_{2} \mathrm{I}_{346}-\text { Berl. AG. Z. }=+0.09 \text {-0."6 } \\
& \text { Par. } 14287 \text { - Berl. AG. Z. }=+0.10+2: 3 \text {. }
\end{aligned}
$$

\begin{tabular}{|c|c|c|c|c|}
\hline * & $\alpha$ & & $\delta$ & Autorità \\
\hline 9 & I $x^{h} 40^{m} 33^{s} \cdot 47$ & $+2 I^{\circ}$ & $54^{\prime}$ I 6". 2 & BB.VI $+22^{\circ} .2401$ \\
\hline 10 & II $42 \quad 28.09$ & +20 & $48 \quad 49.1$ & Berl. AG.Z. $227,23 \mathrm{I}$ \\
\hline I I & $1144 \quad 3^{6.27}$ & +20 & $52 \cdot 54 \cdot I$ & Berl. AG. Z, 58,60, 62 \\
\hline I 2 & 629.24 & +14 & $36 \quad 47 \cdot 5$ & $W_{2} \times 2^{h}: 92$ \\
\hline I 3 & 632.26 & +14 & $39 \quad 58.9$ & $W_{2} \geq 2^{h} \cdot 94$ \\
\hline 14 & $5 \quad 47.94$ & +14 & $8 \quad 52.9$ & $W_{1} \mathrm{I}^{\mathrm{h}} \cdot 4 \mathrm{r}$ \\
\hline I 5 & $83^{6.7^{6}}$ & +12 & 4433.0 & Schj. 4415 \\
\hline
\end{tabular}

Padova, R. Osservatorio, I 893 Agosto.

* 8. BB. $2396-$ Berl. AG.Z. $=+0.17+1.6$.

* 10. W 794 - Berl. AG. Z. $=+1.04+3.7$ Br.-Auw. - Berl. AG. Z. $=+0$ !r $4-0 " 3$.

* I I. W 841.842 - Berl. AG.Z. $=+0.13+\mathrm{I}^{\prime \prime} 7$

\begin{tabular}{|c|c|c|c|c|c|c|c|c|c|c|c|c|c|c|c|}
\hline \multicolumn{2}{|c|}{1893} & \multirow{2}{*}{ M. Z. Kön. } & \multirow{2}{*}{$\frac{\Delta \alpha}{+4^{m} 49^{5} \cdot 93}$} & \multirow{2}{*}{$\frac{\Delta \delta}{-\quad 2^{\prime} 44^{\prime \prime} 7}$} & \multirow{2}{*}{$\frac{\mathrm{Vgl} .}{4.4}$} & \multicolumn{2}{|r|}{$\alpha$ app. } & \multirow{2}{*}{$\frac{\log p . \Delta}{8.960}$} & \multicolumn{3}{|c|}{$\delta$ app: } & \multirow{2}{*}{$\begin{array}{c}\log p .4 \\
0.934\end{array}$} & \multicolumn{2}{|c|}{ Red. ad 1. app. } & \\
\hline 1 li & 10 & & & & & & $28^{\mathrm{m}} \quad 0.66$ & & $+47^{\circ}$ & & & & +0.55 & +8.2 & \\
\hline & Io & 131717 & $+60.5^{2}$ & - & 4.0 & 8 & 2911.25 & $8.038_{n}$ & & - & & - & +0.15 & - & \\
\hline & I I & $12 \quad 1828$ & $-3 \quad 54.95$ & - & 4.0 & 9 & $\circ 45.85$ & $9.2 \times 3$ & & - & & - & $3 \dot{I}$ & - & \\
\hline & I I & $12 \quad 48 \quad 14$ & - & +3 & 0.6 & & - & - & +45 & 18 & 56.6 & 0.9 & - & +8.3 & \\
\hline & $\times 5$ & $10 \quad 58 \quad 27$ & $-526.7 \mathrm{I}$ & +549.7 & 8.4 & ro & 3.65 & 9.589 & 6 & 22 & 5 & 6 & 66 & & \\
\hline & I 5 & II 718 & - I $\quad 11.95$ & +029.1 & 4.8 & 10 & 2928.53 & $9 \cdot 548$ & +36 & 19 & 6 & 0.871 & +0.65 & +6.5 & \\
\hline & 17 & $10 \quad 1242$ & -355.79 & - & 8.0 & Io & $54 \quad 53.20$ & 9.590 & & - & & - & +0.73 & - & \\
\hline & I 7 & II II 40 & - & +254.2 & 0.4 & & - & - & $+3^{2}$ & 3 & 44.6 & 0.872 & - & $+5 . I$ & \\
\hline & 19 & $10355^{1}$ & +347.27 & +1954.0 & $3 \cdot 3$ & II & 1324.90 & 9.566 & +28 & 29 & 9.4 & $0.85 I$ & +0.74 & +4.0 & \\
\hline & I9 & 10574 & $+052.4^{8}$ & $+\mathrm{I} 1.8$ & 4.4 & & 1332.00 & 9.551 & +28 & 27 & 49.1 & 0.864 & +0.76 & +4.1 & \\
\hline & 23 & 10 543 & - & $+\quad 10.2$ & 0.4 & & - & - & +22 & 59 & 20.1 & $0.8_{4} \mathrm{I}$ & 一 & +2.2 & \\
\hline
\end{tabular}

* 12. Lal. 22932 - Schj. $=+0.56+3.6$.

Ringmikrometer-Beobachtungen des Cometen 1893...(Rordame-Quénisset)

angestellt am Heliometer der Sternwarte zu Königsberg von Dr. Fritz Cohn.

\section{Bemerkungen.}

Am ersten Abend (Juli ro) konnte der Comet der hellen Dämmerung und seines tiefen Standes im Norden wegen trotz seiner Helligkeit $\left(3^{\text {ter }}\right.$ bis $4^{\text {ter }} \mathrm{Gr}$.) erst gegen I I Uhr Abends aufgefunden werden; später wurden die Beobachtungen durch den tiefen Stand im Nordwesten insofern beeinträchtigt, als bisweilen kein günstiger Ver- gleichstern (Sterne 8.5 ${ }^{\text {ter }}$ Grösse waren nur mühsam zu beobachten) aufzufinden war.

Juli I I. Luft ganz undurchsichtig; vor Schluss der Beobachtung verschwinden Comet und Stern (7.3 $3^{\text {ter }}$ Gr., dieser zuerst) im Dunst; $\delta$ sehr unsicher.

Juli I 5 und 19. Comet ist zwar schon um $9 \frac{1}{2} \mathrm{Uhr}$, 